\title{
Magnetic activity and differential rotation in the very young star KIC 8429280
}

\author{
A. Frasca ${ }^{1}$, H.-E. Fröhlich ${ }^{2}$, A. Bonanno ${ }^{1}$, G. Catanzaro ${ }^{1}$, K. Biazzo ${ }^{3,1}$, and J. Molenda-Żakowicz ${ }^{4}$ \\ 1 INAF, Osservatorio Astrofisico di Catania, via S. Sofia, 78, 95123 Catania, Italy \\ e-mail: antonio.frasca@oact.inaf.it \\ 2 Leibniz Institute for Astrophysics Potsdam (AIP), An der Sternwarte 16, 14482 Potsdam, Germany \\ 3 INAF, Osservatorio Astronomico di Capodimonte, via Moiariello, 16, 80131 Napoli, Italy \\ 4 Astronomical Institute, Wrocław University, ul. Kopernika 11, 51-622 Wrocław, Poland
}

Received 30 March 2011 / Accepted 3 June 2011

\section{ABSTRACT}

\begin{abstract}
Aims. We present a spectroscopic and photometric analysis of the rapid rotator KIC 8429280, discovered by ourselves as a very young star and observed by the NASA Kepler mission, designed to determine its activity level, spot distribution, and differential rotation. Methods. We use ground-based data, such as high-resolution spectroscopy and multicolor broad-band photometry, to derive stellar parameters $\left(v \sin i\right.$, spectral type, $T_{\text {eff }}, \log g$, and $\left.[\mathrm{Fe} / \mathrm{H}]\right)$, and we adopt a spectral subtraction technique to highlight the strong chromospheric emission in the cores of hydrogen $\mathrm{H} \alpha$ and $\mathrm{Ca}$ II $\mathrm{H} \& \mathrm{~K}$ and infrared triplet (IRT) lines. We then fit a robust spot model to the high-precision Kepler photometry spanning 138 days. Model selection and parameter estimation is performed in a Bayesian manner using a Markov chain Monte Carlo method.

Results. We find that KIC 8429280 is a cool (K2 V) star with an age of about $50 \mathrm{Myr}$, based on its lithium content, that has passed its $\mathrm{T}$ Tau phase and is spinning up approaching the ZAMS on its radiative track. Its high level of chromospheric activity is clearly indicated by the strong radiative losses in $\mathrm{Ca}$ II $\mathrm{H} \& \mathrm{~K}$ and IRT, $\mathrm{H} \alpha$, and $\mathrm{H} \beta$ lines. Furthermore, its Balmer decrement and the flux ratio of Ca II IRT lines imply that these lines are mainly formed in optically-thick regions similar to solar plages. The analysis of the Kepler data uncovers evidence of at least seven enduring spots. Since the star's inclination is rather high - nearly $70^{\circ}-$ the assignment of the spots to either the northern or southern hemisphere is not unambiguous. We find at least three solutions with nearly the same level of residuals. Even in the case of seven spots, the fit is far from being perfect. Owing to the exceptional precision of the Kepler photometry, it is not possible to reach the noise floor without strongly enhancing the degrees of freedom and, consequently, the non-uniqueness of the solution. The distribution of the active regions is such that the spots are located around three latitude belts, i.e. around the star's equator and around $\pm\left(50^{\circ}-60^{\circ}\right)$, with the high-latitude spots rotating slower than the low-latitude ones. The equator-to-pole differential rotation $\mathrm{d} \Omega \simeq 0.27 \mathrm{rad} \mathrm{d}^{-1}$ is at variance with some recent mean-field models of differential rotation in rapidly rotating main-sequence stars, which predict a much smaller latitudinal shear. Our results are consistent with the scenario of a higher differential rotation, which changes along the magnetic cycle, as proposed by other models.
\end{abstract}

Key words. stars: activity - starspots - stars: rotation - stars: chromospheres - stars: individual: KIC 8429280 - X-rays: stars

\section{Introduction}

Starspots are characteristics of solar-like activity observed in cool stars. They are tracers of magnetic flux tube emergence and can provide information about the different forces acting on the flux tubes during their buoyant rise, and on photospheric motions, such as the latitudinal drift of spots during the activity cycle and the differential rotation, which are both essential ingredients of mean field dynamo theory.

However, even though the activity cycle in Sun-like stars is believed to be driven by the meridional circulation with a low eddy diffusivity (Bonanno et al. 2002), the situation is much less clear for late-type rapidly rotating stars. In this latter case, numerical simulations suggest that the magnetic dynamo action can produce wreaths of strong toroidal magnetic field at low

* Based on public Kepler data, on observations made with the Italian Telescopio Nazionale Galileo operated on the island of La Palma by the Fundación Galileo Galilei of INAF (Istituto Nazionale di Astrofisica) at the Spanish Observatorio del Roque del los Muchachos of the Instituto de Astrofisica de Canarias, and on observations collected at the Catania Astrophysical Observatory (Italy). latitudes, often of opposite polarity in the two hemispheres (Nelson et al. 2011). On the other hand, theoretical models of generation of magnetic fields in fully convective pre-main sequence (PMS) stars argue that the basic dynamo action is in the $\alpha^{2}$ regime (Küker \& Rüdiger 1999).

The differential rotation pattern is expected to be dictated by the Taylor-Proudman theorem, so that the iso-contour lines are cylinder-shaped.

Recent calculations of a $1-M_{\odot}$ rapidly rotating mainsequence star with period $P=1.33$ days, predict a value of $0.08 \mathrm{rad} \mathrm{d}^{-1}$, surprisingly close to the solar value despite there being a factor of 20 between the average rotation rates (Küker et al. 2011). However, for HD 171488, a young Sun with an equatorial rotation period of 1.33 days, values of differential rotation as high as $0.50 \mathrm{rad} \mathrm{d}^{-1}$ have been found (e.g., Marsden et al. 2006; Jeffers \& Donati 2008).

A unique opportunity for trying to solve these open problems is given by the NASA Kepler mission, which is providing an unprecedented data set of the photometric variability of a large star sample (Borucki et al. 2010). Its combination of very high photometric precision and long, uninterrupted time coverage is 
essential not only for its primary goal (the discovery of transiting exoplanets) but also for the study of many astrophysical phenomena in stellar variability, such as the magnetic activity and differential rotation in cool stars.

Single active stars in the field can be most efficiently selected on the basis of their high coronal emission, and turn out to be mostly young stars with an age of a few hundred Myr, i.e. in the zero-age main sequence (ZAMS), or even younger, namely weak T Tauri (wTTS) or post-T Tauri (pTTS) stars (see, e.g., Guillout et al. 2009, and reference therein).

KIC 8429280 (=TYC 3146-35-1) was selected as an X-ray active star from the cross-correlation of the ROSAT All-Sky Survey (RASS; Voges et al. 1999, 2000) with Tycho and Hipparcos catalogs (Perryman et al. 1997). It is a relatively bright star $(V=9.9 \mathrm{mag})$ and falls in the field of view of Kepler.

\section{Ground-based observations and data reduction}

\subsection{Spectroscopy}

Three spectra of KIC 8429280 were acquired with SARG, the échelle spectrograph at the Italian TNG telescope (La Palma, Spain). The first one was taken on 2007 May 31 within a survey of optical counterparts to X-ray sources (proposal TAC67AOT15/07A). With the yellow grism and a slit width of $0 . ' 8$, this spectrum has a resolution of $R=\lambda / \Delta \lambda \simeq 57000$, covering the spectral range 4600-7900 $\AA$ in 55 échelle orders, with a signalto-noise ratio $S / N \sim 70$. The other two spectra ( $S / N$ ranging from about 60 to 100$)$ were taken, adopting the same slit width, on 2009 Aug. 11 (proposal TAC71-AOT20/09B) with the red and blue grisms and cover the wavelength ranges 5500-11000 and 3600-5100 A, respectively.

Another spectrum of KIC 8429280 with $S / N \sim 50$ was taken at the M. G. Fracastoro station (Serra La Nave, Mt. Etna, $1750 \mathrm{~m}$ a.s.1.) of the Osservatorio Astrofisico di Catania (OAC - Italy) on 2009 July 13. The 91-cm telescope of the OAC was equipped with FRESCO, a fiber-fed échelle spectrograph that covers the spectral range $4300-6800 \AA$ in 20 orders with a resolution $R \simeq 21000$.

For calibration purposes, spectra of radial and rotational velocity standard stars (see Table 1), as well as bias, flat-field, and arc-lamp exposures were acquired at both observatories during each observing run.

The data reduction was performed with the ECHELLE task of the IRAF $^{1}$ package, following standard steps (see, e.g., Catanzaro et al. 2010).

\subsection{Photometry}

CCD images in the Johnson-Cousins $B, V, R_{\mathrm{C}}$, and $I_{\mathrm{C}}$ filters were acquired using the focal-reducer imaging camera with the $91-\mathrm{cm}$ telescope of the OAC on 2010 November 11. Data reduction was carried out following standard steps of overscan region subtraction, master-bias subtraction, and division by average twilight flat-field images. The $B V R_{\mathrm{C}} I_{\mathrm{C}}$ magnitudes were extracted from the corrected images through aperture photometry performed with DAOPHOT using the IDL ${ }^{2}$ routine APER. Standard stars in

\footnotetext{
1 IRAF is distributed by the National Optical Astronomy Observatory, which is operated by the Association of the Universities for Research in Astronomy, inc. (AURA) under cooperative agreement with the National Science Foundation.

2 IDL (Interactive Data Language) is a registered trademark of ITT Visual Information Solutions.
}

Table 1. Radial/rotational velocity standard stars.

\begin{tabular}{lcccc}
\hline \hline Name & Sp. Type & $\begin{array}{c}R V \\
\left(\mathrm{~km} \mathrm{~s}^{-1}\right)\end{array}$ & $\begin{array}{c}v \sin i^{d} \\
\left(\mathrm{~km} \mathrm{~s}^{-1}\right)\end{array}$ & Notes \\
\hline HD 115404 & K2 V & $7.60^{a}$ & 3.3 & $R V$ \\
HD 145675 & K0 V & $\ldots$ & 0.8 & $v \sin i$ \\
HD 157214 & G0 V & $-79.2^{b}$ & 1.6 & $v \sin i$ \\
HD 10700 & G8 V & $-17.1^{b}$ & 0.9 & $v \sin i$ \\
HD 221354 & K1 V & $-25.20^{a}$ & 0.6 & $R V, v \sin i$ \\
HD 32923 & G4 V & $20.50^{a}$ & 1.5 & $R V, v \sin i$ \\
HD 12929 & K2 III & $-14.6^{c}$ & 1.6 & $R V$ \\
HD 187691 & F8 V & $-0.2^{c}$ & 2.8 & $R V$ \\
HD 182572 & G8 IV & $-100.35^{a}$ & 1.9 & $R V, v \sin i$ \\
HD 161096 & K2 III & $-12.53^{a}$ & 2.1 & $R V$ \\
\hline
\end{tabular}

Notes. ${ }^{(a)}$ Udry et al. (1999) . ${ }^{(b)}$ Nordström et al. (2004). ${ }^{(c)}$ Evans (1967). ${ }^{(d)}$ Glebocki \& Gnacinski (2005).

the cluster NGC 7790 (Stetson 2000) were observed to calculate the transformation coefficients to the Johnson-Cousins system. The zero points for the $V$ magnitude and $B-V, V-R$, and $R-I$ colors were determined by the observation of a standard star close to KIC 8429280 (BD+42 3339, Castelaz et al. 1991). Photometric data are summarized in Table 2.

\section{Target characterization from ground-based observations}

\subsection{Astrophysical parameters}

High-resolution spectroscopy permits us to derive most of the fundamental stellar parameters, such as radial $(R V)$ and projected rotational velocity $(v \sin i)$, spectral type, luminosity class, effective temperature $\left(T_{\text {eff }}\right)$, gravity $(\log g)$, and metallicity $([\mathrm{Fe} / \mathrm{H}])$.

Cross-correlation functions (CCFs) were computed with the IRAF task FXCOR to derive both $R V$ and $v \sin i$. For this purpose, as templates we used spectra of slowly rotating $R V$ standard stars listed in Table 1. We averaged the results from individual échelle orders as described, e.g., in Frasca et al. (2010). The CCFs were used for the determination of $v \sin i$ through a calibration of the full-width at half maximum $(F W H M)$ of the CCF peak as a function of the $v \sin i$ of artificially broadened spectra of slowly rotating standard stars (Table 1) acquired with the same setup and in the same observing seasons as KIC 8429280 (see, e.g., Guillout et al. 2009), obtaining a value of $39.3 \pm 2.4 \mathrm{~km} \mathrm{~s}^{-1}$, where the error is the standard deviation of $v \sin i$ values from each individual échelle order.

We measured, within the errors, the same $R V$ in the SARG spectra of KIC 8429280 taken in 2007 and $2009(R V=$ $-33.1 \pm 0.5 \mathrm{~km} \mathrm{~s}^{-1}$, and $-33.1 \pm 0.4 \mathrm{~km} \mathrm{~s}^{-1}$, respectively) and the FRESCO spectrum of $2009\left(R V=-32.2 \pm 0.8 \mathrm{~km} \mathrm{~s}^{-1}\right)$. The constant $R V$ measured by ourselves and the absence of any sign of duplicity in both the CCF and the line spectrum, suggest that KIC 8429280 could be a single star, although more spectra and high angular resolution images are needed to safely exclude the presence of a stellar companion (see Sect. 5.1 for a wider discussion).

We used the ROTFIT code (Frasca et al. 2003, 2006) to evaluate $T_{\text {eff }}, \log g,[\mathrm{Fe} / \mathrm{H}]$, and re-determine $v \sin i$. We adopted, as reference spectra, a library of 185 ELODIE Archive spectra of standard stars well distributed in effective temperature, spectral type, and gravity, and in a suitable range of metallicities (Prugniel \& Soubiran 2001). The SARG spectrum at 
Table 2. Johnson-Cousins photometric data.

\begin{tabular}{lccccccc}
\hline \hline Name & $B$ & $V$ & $R_{\mathrm{C}}$ & $I_{\mathrm{C}}$ & $J^{a}$ & $H^{a}$ & $K_{\mathrm{s}}{ }^{a}$ \\
\hline KIC 8429280 & $10.88(0.04)$ & $9.94(0.04)$ & $9.38(0.05)$ & $8.86(0.06)$ & $8.114(0.021)$ & $7.672(0.031)$ & $7.541(0.018)$ \\
\hline
\end{tabular}

Notes. Uncertainties are in parenthesis. ${ }^{(a)}$ From 2MASS catalog (Cutri et al. 2003).

$R_{\mathrm{SARG}}=57000$ taken with the yellow grism was convolved with a Gaussian kernel of width $W=\lambda \sqrt{1 / R_{\text {ELODIE }}^{2}-1 / R_{\text {SARG }}^{2}} \AA$ to match the resolution of ELODIE spectra $\left(R_{\text {ELODIE }}=42000\right)$. We applied the ROTFIT code to the échelle orders with a fairly $\operatorname{good} S / N$, which cover the range 4600-6800 , in the observed spectrum. For each order, we estimated the stellar parameters of KIC 8429280 as the mean of the parameters of the ten reference stars that most closely resemble the target spectrum, which is quantified by a $\chi^{2}$ measure, and adopted their standard deviation as a measure of the uncertainty. The adopted estimates for the stellar parameters come from a weighted mean of the values for all the individual orders, the weight accounting also for the $\chi^{2}$ (more weight to the most closely fitted or higher $S / N$ orders) and the "amount of information" contained in each spectral region expressed by the total spectral-line absorption. The standard error in the weighted mean was adopted as the uncertainty estimate for the final values of the stellar parameters.

For the $v \sin i$ determination, we ran ROTFIT using as templates a smaller grid of SARG spectra of inactive and slowly rotating standard stars (Table 1 ) acquired with the same instrumental setup as for our target. This allowed us to remove any eventual systematic error in the artificial broadening of template spectra introduced by the different resolution and provided us with a measure of $v \sin i$ independent of the CCF method. The $v \sin i$ determined with ROTFIT, $37.1 \pm 2.9 \mathrm{~km} \mathrm{~s}^{-1}$, agrees with the value derived from the CCF. For these particular tasks, the $\mathrm{H} \alpha$ and $\mathrm{Li}$ I lines, as well as spectral regions affected by telluric features, were excluded from the analyzed spectral range.

We also determined the stellar parameters by fitting selected spectral regions with synthetic spectra generated by SYNTHE (Kurucz \& Avrett 1981) using ATLAS9 (Kurucz 1993) atmosphere models. When applying all the models, we considered a solar opacity distribution function and microturbulence velocity $\xi=1 \mathrm{~km} \mathrm{~s}^{-1}$, which are typical of a star with $T_{\text {eff }}=5000 \mathrm{~K}$ and $\log g=4.5$ (see, e.g., Allende Prieto et al. 2004).

As starting values of $T_{\text {eff }}$ and $\log g$, we used those derived from ROTFIT. At the same time, we determined the projected rotational velocity by matching the metal lines present in our spectral range. We adopted the lists of spectral lines and the atomic parameters from Castelli \& Hubrig (2004), who updated the parameters listed originally by Kurucz \& Bell (1995). The stellar parameters were determined by minimizing the difference between the observed and the synthetic spectra, using the $\chi^{2}$ as the goodness-of-fit parameter. Errors were estimated to be the variation in the parameters that increases the $\chi^{2}$ of a unit.

In Fig. 1, we show the result of our fitting of two different wavelength regions containing important neutral iron and calcium lines, which are well suited to constraining temperature, gravity, and metallicity. The cores of the two strong Fe I lines at $\lambda 5227.2$ and $\lambda 5232.9$ are possibly influenced by NLTE effects and chromospheric filling.

In Table 3, we list the stellar parameters derived with both ROTFIT and SYNTHE, which agree very well with each other.

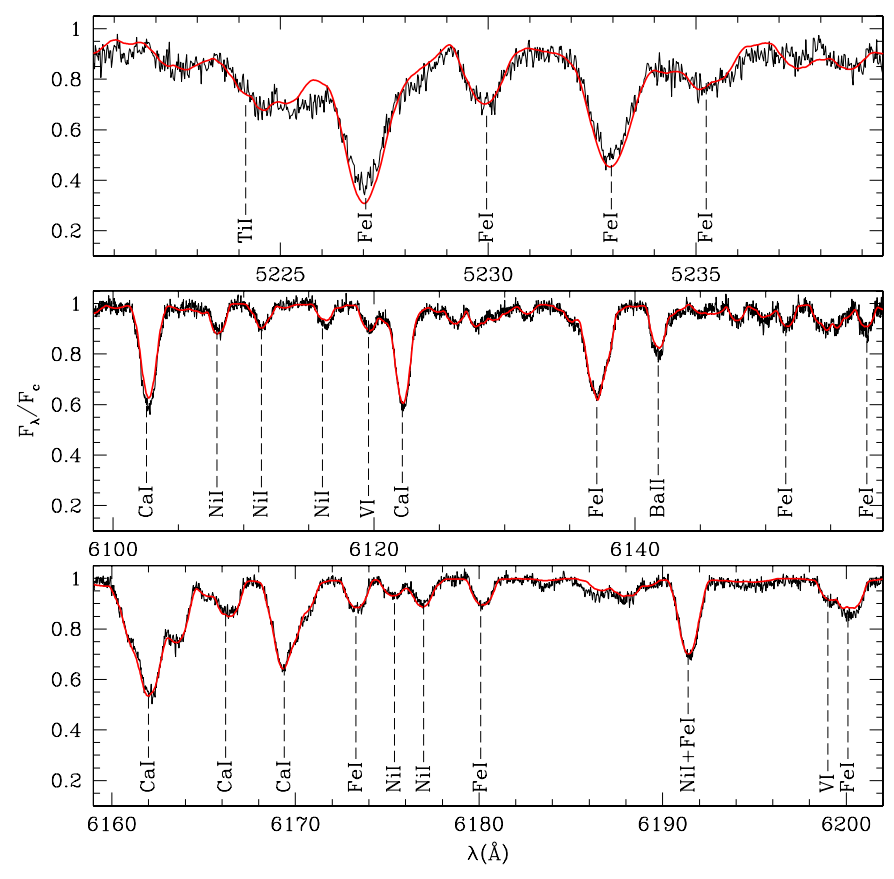

Fig. 1. Continuum-normalized observed spectrum (solid black line) in the region between 5220 and $5240 \AA$ (top panel) and between 6100 and $6200 \AA$ (middle and bottom panels) of KIC 8429280. The synthetic spectrum calculated with the parameters listed in Table 3 is superimposed (smooth red line).

\subsection{Chromospheric activity and lithium abundance}

We evaluated the level of chromospheric activity from the emission in the cores of the Ca II H\&K and IRT lines, as well as in the Balmer $\mathrm{H} \alpha, \mathrm{H} \beta$, and $\mathrm{H} \epsilon$ lines. The star's age was inferred from the lithium abundance. We estimated both the lithium equivalent width and the chromospheric emission level with the "spectral subtraction" technique (see, e.g., Herbig 1985; Frasca \& Catalano 1994). This technique is based on the subtraction of a "non-active", "lithium-poor" template made with observed spectra of slowly rotating stars of the same spectral type as KIC 8429280 with a negligible level of chromospheric activity and no apparent lithium absorption line. The equivalent width of the lithium line $\left(W_{\mathrm{Li}}\right)$ and the net equivalent width of the Ca II H\&K, Ca II IRT, $\mathrm{H} \alpha$, and $\mathrm{H} \beta$ lines $\left(W^{\mathrm{em}}\right)$ were measured, in the spectrum obtained after subtracting from the target the nonactive template, by integrating the residual emission (absorption for the lithium line) profile.

The $\mathrm{Ca}$ II $\mathrm{H} \& \mathrm{~K}$ lines display strong emission cores that appear nearly symmetric without any evidence of self-absorption. The $\mathrm{H} \epsilon$ emission is clearly visible and becomes more evident after the subtraction of the non-active template (Fig. 2). The peak intensity of the $\mathrm{H} \& \mathrm{~K}$ lines is similar to that displayed by SAO 51891, a very young K0-1 star studied by Biazzo et al. (2009). 
Table 3. Astrophysical parameters.

\begin{tabular}{|c|c|c|c|c|c|c|c|c|}
\hline Name & Sp. Type & $\begin{array}{c}R V \\
\left(\mathrm{~km} \mathrm{~s}^{-1}\right)\end{array}$ & $\begin{array}{l}T_{\text {eff }} \\
(\mathrm{K})\end{array}$ & $\log g$ & {$[\mathrm{Fe} / \mathrm{H}]$} & $\begin{array}{c}v \sin i \\
\left(\mathrm{~km} \mathrm{~s}^{-1}\right)\end{array}$ & $\begin{array}{l}W_{\mathrm{Li}} \\
(\mathrm{m} \AA)\end{array}$ & $\log N(\mathrm{Li})$ \\
\hline KIC 8429280 & $\mathrm{~K} 2 \mathrm{~V}$ & $-33.1(0.5)$ & $\begin{array}{l}5055(135)^{a} \\
5000(100)^{b}\end{array}$ & $\begin{array}{l}4.41(0.25)^{a} \\
4.50(0.10)^{b}\end{array}$ & $\begin{array}{l}-0.02(0.10)^{\mathrm{a}} \\
-0.05(0.10)^{\mathrm{b}}\end{array}$ & $\begin{array}{l}37(3)^{a} \\
39(3)^{b}\end{array}$ & $270(20)$ & $2.9(0.1)$ \\
\hline
\end{tabular}

Notes. Uncertainties are in parenthesis. ${ }^{(a)}$ From ROTFIT. ${ }^{(b)}$ From SYNTHE.
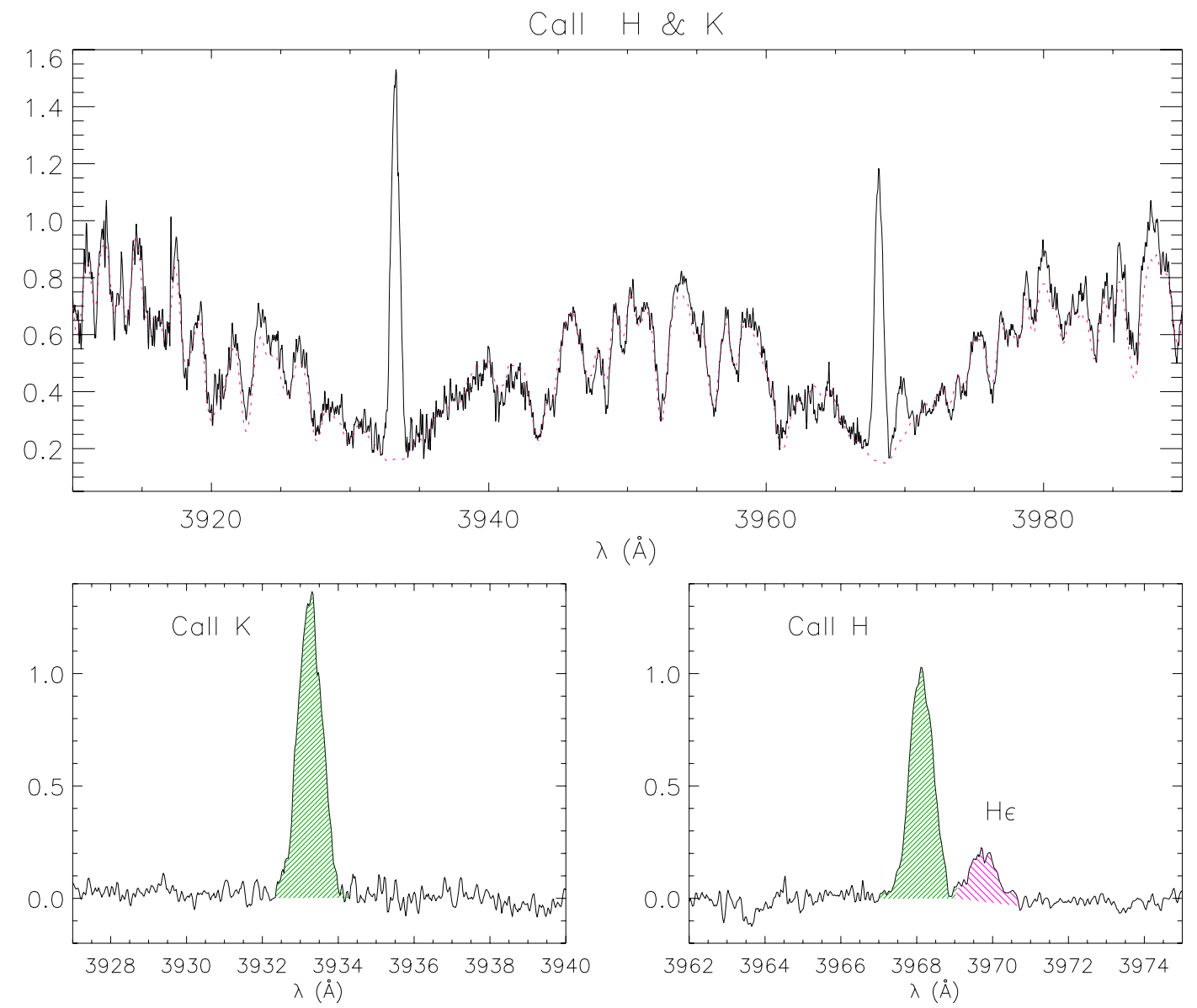

Fig. 2. Top panel: continuum-normalized SARG spectrum of KIC 8429280 (solid line) in the Ca II H \& K region together with the inactive stellar template (dotted red line) broadened at the $v \sin i$ of the target and Doppler-shifted according to the $R V$ difference. Bottom panels: the difference spectrum. $\mathrm{H} \epsilon$ emission, already apparent in the observed spectrum, is clearly emphasized in the residuals.
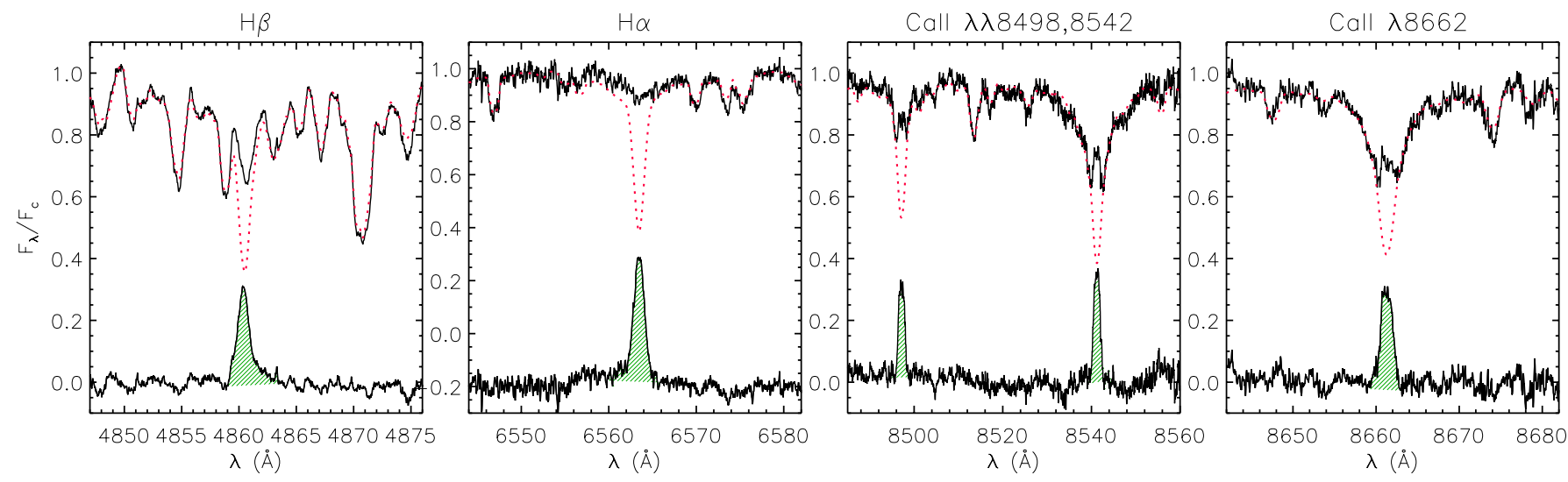

Fig. 3. Top of each panel: observed, continuum-normalized SARG spectra of KIC 8429280 (solid line) in the H $\beta$, H $\alpha$, and Ca II IRT regions together with the non-active stellar template (dotted red line). Bottom of each panel: difference between observed and template spectra. The residual $\mathrm{H} \alpha$ profile is plotted shifted downwards by 0.2 for the sake of clarity. The hatched areas represent the excess emissions that have been integrated to get the net equivalent widths. 
Table 4. Line equivalent widths and radiative chromospheric losses.

\begin{tabular}{|c|c|c|c|c|}
\hline Line & $\begin{array}{c}\text { Date } \\
(\text { yyyy } / \mathrm{mm} / \mathrm{dd})\end{array}$ & $\begin{array}{l}W^{\mathrm{em}} \\
(\AA)\end{array}$ & $\begin{array}{c}\text { Error } \\
(\AA)\end{array}$ & $\begin{array}{c}\text { Flux } \\
\left(\operatorname{erg~cm}{ }^{-2} \mathrm{~s}^{-1}\right)\end{array}$ \\
\hline $\mathrm{H} \alpha$ & $2007 / 05 / 31$ & 0.922 & 0.107 & $3.35 \times 10^{6}$ \\
\hline " & $2009 / 07 / 13$ & 1.026 & 0.150 & $3.73 \times 10^{6}$ \\
\hline " & $2009 / 08 / 11$ & 0.999 & 0.071 & $3.63 \times 10^{6}$ \\
\hline $\mathrm{H} \beta$ & $2009 / 07 / 13$ & 0.315 & 0.118 & $1.16 \times 10^{6}$ \\
\hline " & $2009 / 08 / 11$ & 0.442 & 0.076 & $1.63 \times 10^{6}$ \\
\hline $\mathrm{H} \epsilon$ & $" \prime \prime$ & 0.264 & 0.040 & $0.48 \times 10^{6}$ \\
\hline $\mathrm{Ca}$ II $\mathrm{H}$ & " " & 1.075 & 0.068 & $1.66 \times 10^{6}$ \\
\hline Ca II $\mathrm{K}$ & $" 1$ & 0.795 & 0.045 & $1.45 \times 10^{6}$ \\
\hline Са II IRT $\lambda 8498$ & " " & 0.538 & 0.065 & $1.45 \times 10^{6}$ \\
\hline Ca II IRT $\lambda 8542$ & $" 1$ & 0.624 & 0.065 & $1.69 \times 10^{6}$ \\
\hline Ca II IRT $\lambda 8662$ & & 0.596 & 0.074 & $1.63 \times 10^{6}$ \\
\hline
\end{tabular}

In Fig. 3, the spectral subtraction reveals Ca II IRT lines with emission reversals in their cores and a remarkable filling-in of the $\mathrm{H} \beta$ line. Furthermore, the $\mathrm{H} \alpha$ line is completely filled-in by emission, with high values of net equivalent width (see Table 4). This behavior is typical of very active stars and the $\mathrm{H} \alpha$ profile is quite similar to those frequently displayed by LQ Hya, a well-studied young star for which the $\mathrm{H} \alpha$ changes from filledin to weak emission profiles (Fekel et al. 1986; Strassmeier et al. 1993; Frasca et al. 2008). The similarity between KIC 8429280 and LQ Hya goes beyond the $\mathrm{H} \alpha$ profile, since the spectral type is the same for both stars $(\mathrm{K} 2 \mathrm{~V})$, the effective temperature is nearly identical, and the rotation period of LQ Hya is only slightly longer (1.6 versus 1.2 days).

We also evaluated the total radiative losses in the chromospheric lines following the guidelines of Frasca et al. (2010), i.e. by multiplying the average $W^{\mathrm{em}}$ by the continuum surface flux at the wavelength of the line. The latter was evaluated by means of the spectrophotometric atlas of Gunn \& Stryker (1983) and the angular diameters calculated by applying the Barnes \& Evans (1976) relation. The net equivalent widths and the chromospheric line fluxes are reported in Table 4.

On the basis of the $\mathrm{H} \alpha$ and $\mathrm{H} \beta$ flux, we evaluated a Balmer decrement $F_{\mathrm{H} \alpha} / F_{\mathrm{H} \beta}$ in the range 2.2-3.2. Values of the Balmer decrement in the range 1-2 are typical of optically thick emission by solar and stellar plages (e.g., Chester 1991; Buzasi 1989), while prominences seen off-limb give rise to values of 10. The flux ratio of two Ca II-IRT lines, $F_{8542} / F_{8498}=1.2$, indicative of high optical depths, is in the range of the values found by Chester (1991) in solar plages. Solar prominences have instead values of $F_{8542} / F_{8498} \sim 9$, typical of an optically-thin emission source.

This suggests that the bulk of chromospheric emission of KIC 8429280, in both the Balmer and the Ca II lines, is basically due to magnetic surface regions similar to solar plages, and eventually prominences will play a marginal role.

The subtraction method also permits the measurement of the lithium equivalent width cleaned up from the contamination of the close Fe I $\lambda 6707.4 \AA$ photospheric line (Fig. 4). The lithium equivalent width, $W_{\mathrm{Li}}=270 \pm 20 \mathrm{~m} \AA$, above the Pleiades (100 Myr) upper envelope (Soderblom et al. 1993), translates into a very high lithium abundance, $\log N(\mathrm{Li}) \simeq 2.9 \pm 0.1$, adopting the calibrations proposed by Pavlenko \& Magazzù (1996). With this lithium abundance and $T_{\text {eff }}=5055 \mathrm{~K}$, the star falls on the upper envelope of the $\alpha$ Per cluster (50 Myr, Sestito \& Randich 2005), indicating an age of $\approx 50 \mathrm{Myr}$.

For comparison, Fekel et al. (1986) measured for LQ Hya a slightly lower lithium equivalent width, $W_{\mathrm{Li}}=234 \mathrm{~m} \AA$,

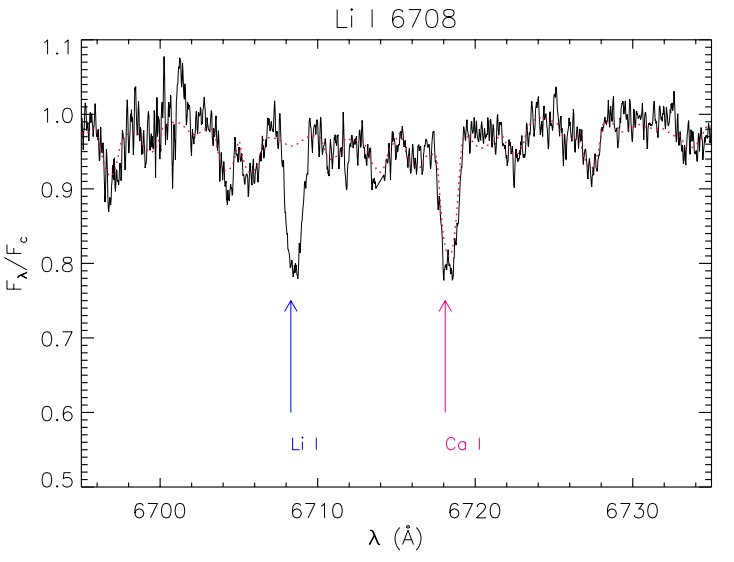

Fig. 4. Continuum-normalized spectrum (solid black line) in the lithium region of KIC 8429280 together with the template spectrum (dotted red line) of the non-active lithium-poor reference star, broadened at the $v \sin i$ of the target and Doppler-shifted according to the $R V$ difference.

deducing a lithium abundance $\log N(\mathrm{Li}) \simeq 2.8$, and concluded that star should be at least as young as the Pleiades. This supports the measurement of a very young age for KIC 8429280, which could still be in the wTTS or pTTS phase and not yet have reached the ZAMS.

\subsection{Spectral energy distribution}

The standard $B V R_{\mathrm{C}} I_{\mathrm{C}}$ photometry (Table 2), complemented with $J H K_{\mathrm{s}}$ magnitudes from the 2MASS catalog (Cutri et al. 2003), allowed us to reconstruct the spectral energy distribution (SED) from the optical to the near infrared (IR) domain.

To perform a fit to the SED, we used the grid of NextGen low-resolution synthetic spectra (Hauschildt et al. 1999) with $\log g=4.0$ and 4.5 and solar metallicity. The effective temperature was set to $5000 \mathrm{~K}$ in agreement with the value found with ROTFIT and SYNTHE (Table 3). The interstellar extinction $\left(A_{V}\right)$ was evaluated from the distance according to the rate of $0.8 \mathrm{mag} / \mathrm{kpc}$ found by Mikolajewska \& Mikolajewski (1980) for the sky region around CI Cyg (very close to our target). Finally, the angular diameter $(\phi)$, which scales the model surface flux over the stellar flux at Earth, was allowed to vary. The Cardelli et al. (1989) extinction law with $R_{V}=3.1$ was used. The best-fit solution was found by minimizing the $\chi^{2}$ of the fit to the $B V R_{\mathrm{C}} I_{\mathrm{C}} J$ data, which are dominated by the photospheric flux of the star and are normally not appreciably affected by infrared excesses. The angular diameter derived from the SED, $\phi=0.140$ mas, implies a distance $d \simeq 50$ pc if we adopt a ZAMS radius of $0.75 R_{\odot}$. Adopting a radius of $0.88 R_{\odot}$, which is typical of a 50-Myr old star with the same temperature as KIC 8429280 (see Sect. 4.1), a distance of about $60 \mathrm{pc}$ is derived.

As seen in Fig. 5, the SED is quite well reproduced by the synthetic spectrum and no excess is visible at near-IR wavelengths.

This provides a further check of the effective temperature and assures that this star has by far exceeded the $\mathrm{T}$ Tau phase, largely dissipating its accretion disk. However, a thinner "debris disk", whose presence could be only detected by mid- or far-IR data (lacking for KIC 8429280), cannot be excluded.

\section{Kepler light curve and spot modeling}

\subsection{Photometric data}

We analyzed the long-cadence data of quarter $0(\mathrm{Q} 0)$ to quarter 2 (Q2). The 6174 data points are taken from 2009 May 2 to 


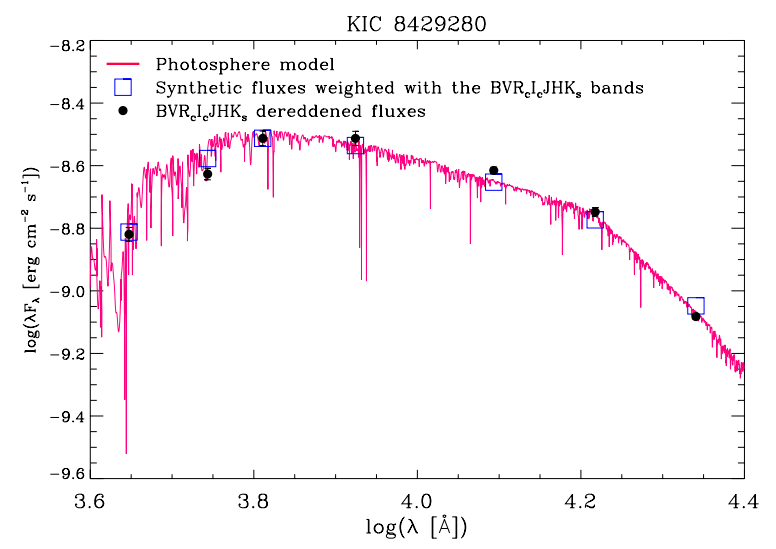

Fig. 5. Spectral energy distribution for KIC 8429280 (dots). The NextGen spectrum with $T_{\text {eff }}=5000 \mathrm{~K}$ and $\log g=4.0$ is overplotted with a continuous line.

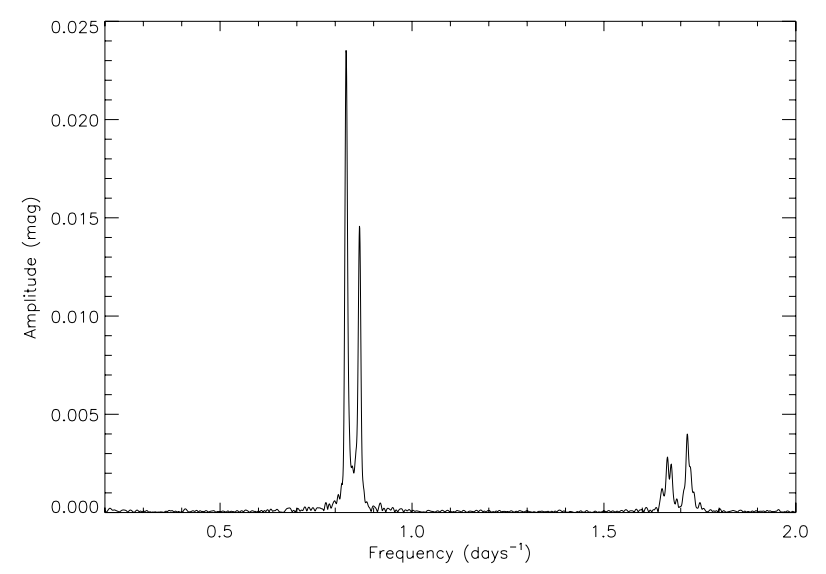

Fig. 6. Power spectrum of the Kepler $\mathrm{Q} 0+\mathrm{Q} 1+\mathrm{Q} 2$ time series of KIC 8429280.

2009 September 16, spanning nearly 138 days with a sampling of one photometric point each $30 \mathrm{~min}$. The most pronounced gap, between Q1 and Q2, amounts to about 4.5 days.

The power spectrum of the Kepler time series, displayed in Fig. 6, shows two main peaks very close in frequency $(0.828$ and $0.863 \mathrm{~d}^{-1}$ ), which correspond to periods of about 1.207 and 1.159 days, respectively. The period uncertainty, estimated from the FWHM of the spectral window, is about 0.006 days. The two low-amplitude peaks at frequency of $\approx 1.66$ and $1.72 \mathrm{~d}^{-1}$ are overtones of the two main peaks.

The double-peaked periodogram is a clear fingerprint of differential rotation, as shown, e.g., by Lanza et al. (1994) in their simulation of light curves of differentially rotating spotted stars. They show that a Fourier analysis of uninterrupted time series of photometry with high precision $\left(\frac{\Delta F}{F}=10^{-5}-10^{-6}\right.$, which is comparable with the precision of the Kepler data) may detect a Sun-like latitudinal differential rotation.

A parameter needed to apply a spot model to our data is the inclination of the rotation axis with respect to the line of sight. From the values of $v \sin i$, stellar radius $R$, and rotation period $P$ the inclination of the rotation axis can be estimated as

$\sin i=\frac{v \sin i P}{2 \pi R}$.

The stellar radius cannot be estimated from effective temperature and luminosity, because this star has no Hipparcos parallax and the Tycho value is unreliable. From Eq. (1), a lower limit follows, $0.85 R_{\odot}$, which exceeds the radius of a ZAMS star with
$T_{\text {eff }}=5055 \mathrm{~K}$ and a mass of $0.76 M_{\odot}, R \simeq 0.75 R_{\odot}$. Thus, we considered a radius of a younger star at the same temperature. We found, from the evolutionary tracks of Siess et al. (2000), that a $\sim 50 \mathrm{Myr}$ star of $0.9 \mathrm{M}_{\odot}$ has the same temperature of our target, but a radius of $0.88 R_{\odot}$. With this value, we find $\sin i \simeq 0.965$, which implies an inclination of about $75^{\circ}$. Such a high inclination is confirmed by the value of $i \simeq 70^{\circ}$ derived from the light-curve solution (see Sect. 4.3).

\subsection{Bayesian photometric imaging}

Photometric imaging can be a many parameter problem. An efficient and robust method is light-curve fitting using circular spots. We chose to use Dorren's (1987) analytical starspot model in its generalization to a quadratic limb-darkening law. Any departure from the spherical shape caused by the star's fast rotation was neglected.

In the case of seven spots, we have 46 free parameters. Three parameters describe the star: inclination angle $i$, equatorial angular velocity $\Omega_{\text {eq }}$, and equator-to-pole differential rotation $\mathrm{d} \Omega$. To reduce the number of free parameters and enforce a monotonic dependence on latitude, $\beta$, differential rotation is prescribed by a Sun-like $\sin ^{2}$-law:

$\Omega(\beta)=\Omega_{\mathrm{eq}}-\mathrm{d} \Omega \sin ^{2} \beta$.

An additional parameter describes the spot rest intensity $\kappa$. For each spot, six parameters are needed: the longitudes in an arbitrarily rotating reference system at the beginning and at the end of the observation, the spot area at a given time, and three further parameters describing spot area evolution (for details see below). The two coefficients $u^{+}$and $u^{-}$in the quadratic limb-darkening law (cf. Alonso et al. 2008) were fixed using the tabulated coefficients from stellar model atmosphere calculations (Sing 2010): $u^{+}=0.7042$ and $u^{-}=0.3774$. The limb darkening is assumed to be the same for the unperturbed photosphere as well as for the spots.

The parameterized law of differential rotation (Eq. (2)) provides the absolute value of a spot's latitude $\beta$ from its rotational frequency. To find out the hemisphere to which a spot belongs, we made a test run with 128 Markov chains in parallel, trying out all possibilities to arrange seven spots in the two hemispheres. Since the inclination was allowed to take negative values, there are always two Markov chains describing the same spot allocation situation. From this 64-h test run, the three different solutions mentioned below (Sect. 4.3) emerged. It cannot be excluded for certain that there are further solutions.

Spot evolution is described by a simple ansatz: the spot area, expressed in units of the star's cross-section, is assumed to evolve basically linearly with time. For a decaying spot, this prescription allows in principle an estimate of the turbulent magnetic diffusivity. The spot evolution model even allows for one sudden change in the slope of the area-versus-time relation. Hence, altogether four parameters are needed to describe the area of a spot and its evolution: area at the time of the (sharp) bend, this time itself and two time derivatives of the spot area.

In addition to the free parameters of the model, the derived parameters, whose physical meaning is relevant, are considered. We are interested in their marginal distributions, in order to derive the mean values and error bars. Examples are the latitude and period of a spot. The latter simply follows from the initial and final longitude of the spot, the former (except for its sign, i.e. the stellar hemisphere on which the spot is located) from the spot's rotation period and the two coefficients of the $\sin ^{2}$-law for the differential rotation. 


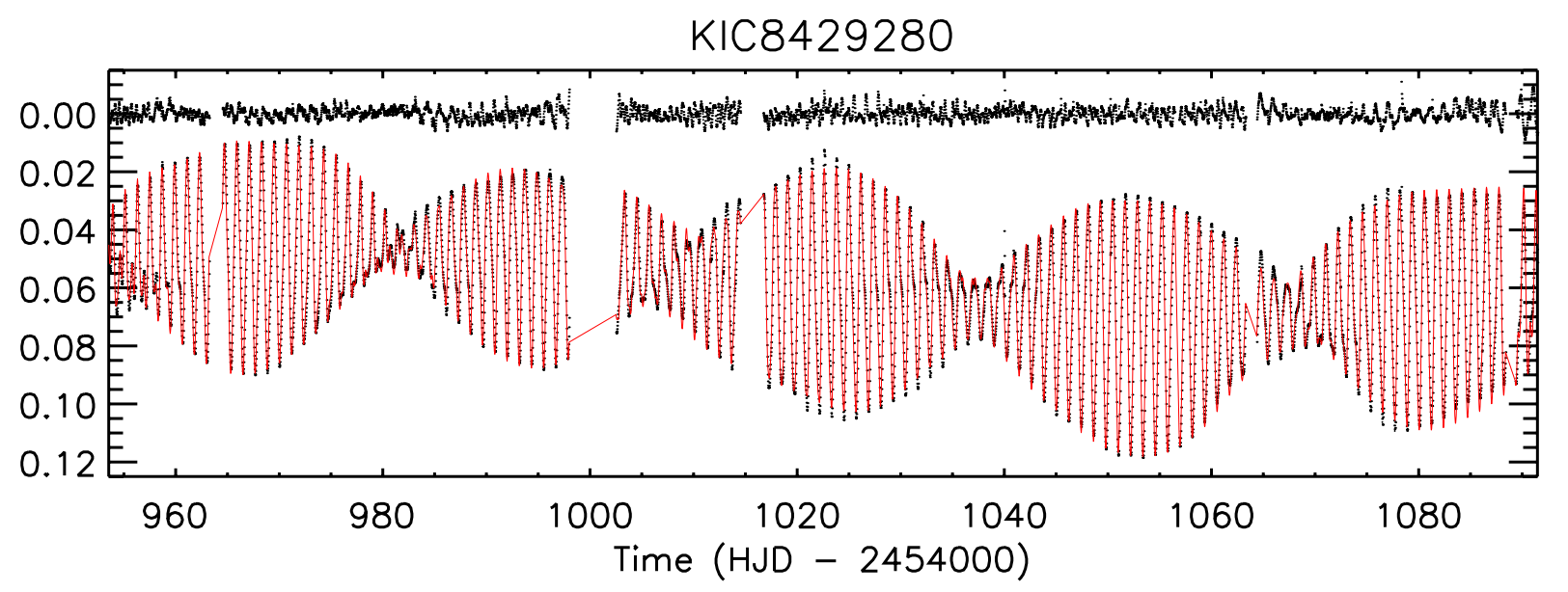

Fig. 7. Robust fit of the Kepler light curves. Q0 is from 953.53825 to 963.24461 , Q1 from 964.51154 to 997.98296 , and Q2 from 1002.76455 to 1091.46678. The solid line is the best fit of the first solution. The residuals, shown at the top, are $\pm 2.36 \mathrm{mmag}$. The gaps are artificially bridged by straight connecting lines.

To estimate all these parameters from the data, a Bayesian method was applied. This provides a straightforward way of estimating parameters and their uncertainties from the data alone. Before analyzing the data, proper prior distributions have to be assigned. If information about the star's inclination $i$ is lacking and selection effects are ignored, $\cos i$ should be evenly distributed for $0 \leq i \leq \pi / 2$. All non-dimensionless parameters such as radii or periods are represented by their logarithms. This ensures that the posterior distribution for a radius will be consistent with that of an area and likewise the posterior for a period with that of a frequency, i.e. it makes no difference whether one prefers radii or areas, periods or frequencies.

The likelihood function is constructed as follows. Spotted stars that are geometrically similar exhibit the same light curve, except for an offset in magnitude. This offset is considered arbitrary and removed by integration. Only the shape of the light curve matters. Hence, it is unnecessary to specify the magnitude of the unspotted star, which is anyhow unknown. It is also assumed that the measurement errors have a Gaussian distribution. The integration over the offset can then be done analytically, which is quite convenient.

With the $N$ data points $d_{i}$ gained at times $t_{i}$, their standard deviation $\sigma$, the fit $f_{0}\left(t_{i}, p_{1} \ldots p_{M}\right)$ and an offset $c_{0}$, the likelihood reads

$$
\begin{aligned}
& \Lambda\left(\sigma, c_{0}, p_{1} \ldots p_{M} ; d_{i}\right)= \\
& \quad \prod_{i=1}^{N} \frac{1}{\sqrt{2 \pi} \sigma} \exp \left(-\frac{\left(d_{i}-f_{0}\left(t_{i}, p_{1} \ldots p_{M}\right)-c_{0}\right)^{2}}{2 \sigma^{2}}\right) .
\end{aligned}
$$

The $M$ unknown parameters are denoted by $p_{j}$, with $j=1 \ldots M$. Integrating analytically away first the measurement error $\sigma$ - using Jeffreys' $1 / \sigma$-prior (cf. Kass \& Wasserman 1996) - and then the uninteresting offset $c_{0}$, one gets a likelihood depending on spot modeling parameters $p_{1} \ldots p_{\mathrm{M}}$ only. It is this mean likelihood, averaged appropriately over measurement error and offset, from which the posterior density distributions for all unknowns are obtained by marginalization. We can argue that the method itself determines error and offset from the data.

When the light curve consists of different parts, first the likelihood of each individual part is determined. Afterwards all likelihoods are multiplied. This means, each part has been assigned its own error level and offset.
We note that, besides an offset, a linear trend in the data may be taken into consideration, where the slope is also integrated away analytically.

The Markov chain Monte Carlo (MCMC) method (cf. Press et al. 2007) has been applied to explore the likelihood mountain in the high-dimensional parameter space. Basically, MCMC is able to find the globally best solution.

\subsection{Results}

There are six gaps exceeding a few hours in the combined light curve. Accordingly, the light curve has been divided into seven parts, with each part being assigned its own offset and error level. Hence, artificial vertical jumps between the data sets do not matter.

The MCMC method found three solutions, all with the same level of residuals. Two of the three solutions are quickly relaxed. The non-relaxed solution needs in any case very dark spots with spot intensity around 0.2 and would lead to a rather high value of the equator-to-pole differential rotation of $0.35 \mathrm{rad} \mathrm{d}^{-1}$. We, therefore, dismiss that solution. Nevertheless, all three solutions show nearly the same spot periods, which means that the maximal observed rotation shear is remarkably the same: $\simeq 0.21 \mathrm{rad} \mathrm{d}^{-1}$. What is different are the latitudes of the spots and the spot evolution. In what follows, we prefer the solution with the lowest degree of differential rotation. All figures refer to that solution if not stated otherwise. Table 5 provides values of some parameters for both relaxed solutions.

A quick glance at the light curve (Fig. 7) reveals the presence of two enduring principal periods. The rotational periods are indeed tightly grouped around $P \simeq 1.16$ and $P \simeq 1.20$ days (Fig. 8) as already indicated by the power spectrum (Fig. 6).

The stellar surface obviously displays a plethora of long lasting features. Within our framework of up to seven dark circular spots, we are unable to fit the light curve down to the measurement errors. At the most, we can fit a seven-spot model to the data, with the residuals being $2.36 \mathrm{mmag}$ rms. The residuals, exceeding by far the measurement errors, are undoubtedly due to the incapability of the model to cope fully with the data.

The gain in goodness-of-fit relative to, e.g., a six-spot model is striking. To give an example, in a first analysis the combined Q0 and Q1 data sets only had been considered. In the case of this 45-day subset, the residuals shrink from 1.26 mmag to 


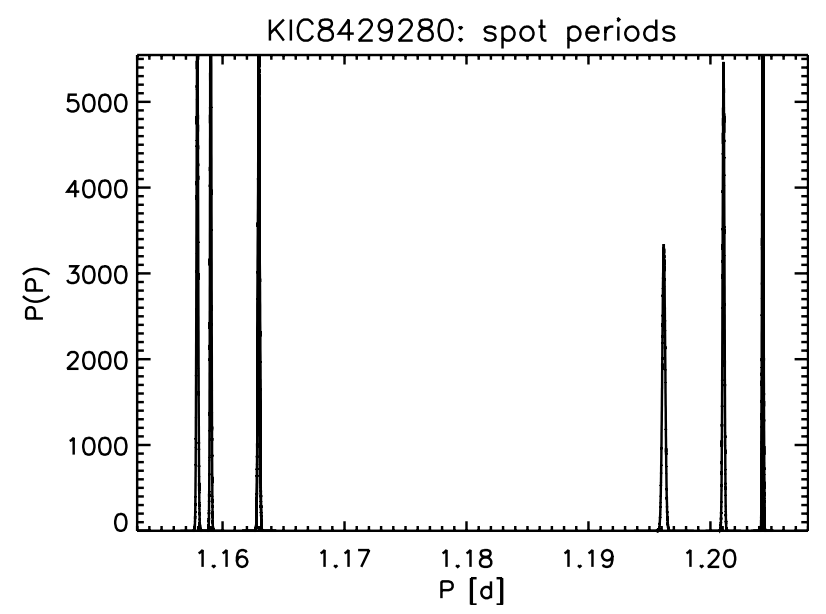

Fig. 8. Marginal distributions of spot periods. The two slowest revolving spots exhibit the same period. The periods group around two principal periods, which produces the obvious "beating" phenomenon quite understandable.

1.04 mmag if one enlarges the number of spots from six to seven. The Bayesian information criterion (BIC) clearly justifies the increase in the number of free parameters. The BIC (Schwarz 1978) allows theory ranking taking into account the goodnessof-fit as well as the number of free parameters used. With $N$, the number of measurements, $\sigma^{2}$, the mean variance in one measurement, and $M$, the number of free parameters, the criterion reads $\mathrm{BIC}=N \log \left(\sigma^{2}\right)+M \log (N)$. It expresses Occam's razor in mathematical terms. A model with a low BIC should preferably be used.

Attempts to reduce the number of free parameters without any loss of information (according to BIC) were in vain. For example, we tried to fit the light curve with two different periods only. In all cases, the loss of goodness-of-fit, as expressed by $\sigma$, outclasses the gain in credibility by far. Thus, one has to accept that - owing to the unprecedented precision of the data - it is impossible to reduce the number of free parameters noticeably. That all these free parameters are well constrained by the data is testified by the mere fact that the Markov chains generally quickly converge to a relaxed solution. On the other hand, we did not try to reduce the size of the residuals of the fit by enhancing the complexity of the spot model (more spots, different spot temperatures, faculae, etc.), because this would require an increase in the number of free parameters with a strong growth of the computing time and a loss of solution uniqueness.

The marginal distribution for the inclination is shown in Fig. 9. The inclination angle is photometrically very well constrained, as is apparent from the smallness of the 68-per-cent confidence region.

The spot intensity, measured in units of the unspotted photosphere, is $\simeq 0.57$ (cf. Fig. 10) which sounds reasonable, because, in the case of sunspots, the bolometric contrast is 0.67 (e.g., Lanza et al. 2003, not distinguishing between umbra and penumbra). For KIC 8429280, a bolometric contrast of 0.57 would translate into a temperature difference of $\simeq 650 \mathrm{~K}$.

The spots are rather large and they slowly evolve as appears in Fig. 11. Despite their large areas, the spots are never really touching each other. Hence, spot evolution does not seem to be influenced by the need to avoid spot overlapping. One should note that the spot evolution ansatz makes sense within the time span of photometric observation only. It must not to be extrapolated to any later times.

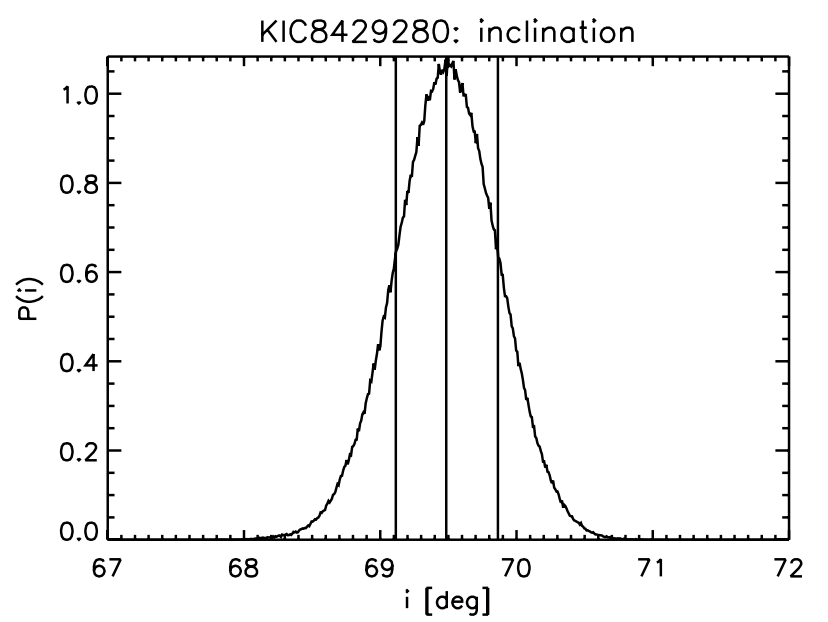

Fig. 9. The star's inclination from Kepler photometry. Vertical lines mark the mean and the 68-per-cent confidence region. The inclination value confirms the relative large radius of the star.

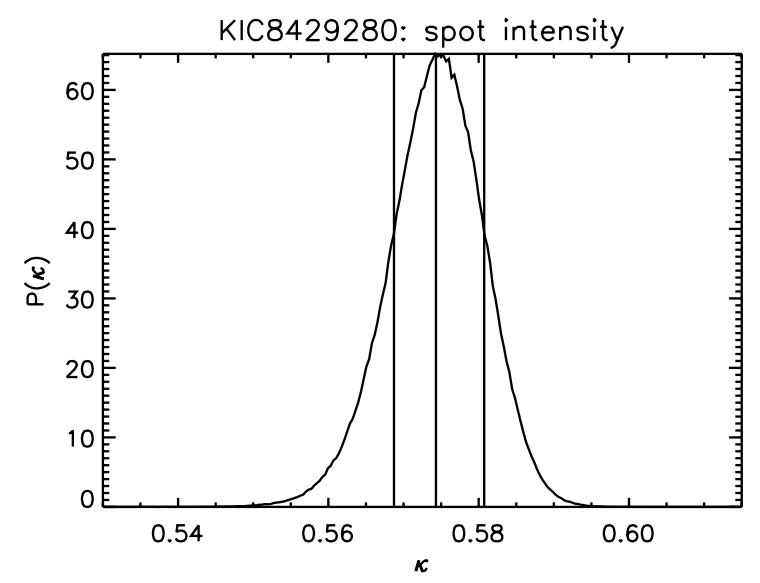

Fig. 10. Spot intensity with respect to the unspotted photosphere. Vertical lines mark the mean and the 68-per-cent confidence region.

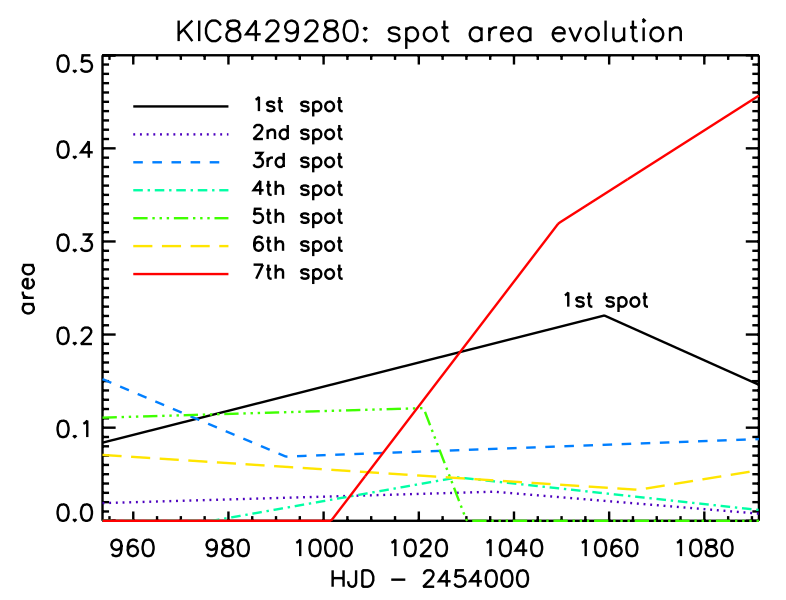

Fig. 11. Evolution of spot areas. Areas are expressed in units of the star's cross-section. The largest spots are those at high latitudes.

As already mentioned, in terms of spot area evolution, the two solutions are quite different. We, therefore, provide for the second solution the corresponding spot area evolution plot, too (Fig. 12).

A major difference between the two solutions concerns spots 5 and 7 . Spot 7 of the first solution (the largest one at 


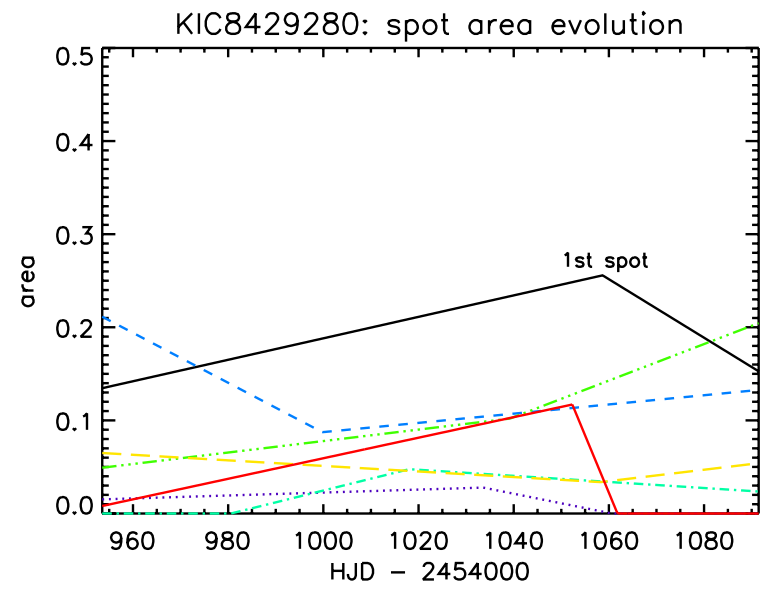

Fig. 12. Evolution of spot areas for the second solution. The different types of line have the same meaning as in Fig. 11.

the end) has partly replaced spot 5 of the second solution. This change in identity is already indicated in the periods. One should also note that there are, apart from spot 3, small but nevertheless highly significant differences in the periods of all the other spots (Table 5).

That there are at least two solutions is not surprising. The inclination is rather high, so it is difficult to determine to which hemisphere a spot belongs.

The main result concerns differential rotation. In the case of KIC 8429280, the spots are obviously long lasting, allowing us to separate the effects of spot evolution from differential rotation. However, even then a cautionary note is in place because of the large number of spots involved. In principle, the MCMC method should be able to find all possible spot periods, in practice one gets at best one feasible set of periods - a conceivable "scenario", not more! The assignment of some dip in the light curve to a certain spot is not unambiguous. We lack the information about what happens when a spot is out of sight.

The marginal distribution of the equator-to-pole differential rotation is given in Fig. 13. For our preferred model, it is $0.266 \pm 0.001 \mathrm{rad} \mathrm{d}^{-1}$, given a $\sin ^{2}$ law as a basis for the latitudinal dependence of differential surface rotation. The second solution results in an even larger differential rotation of $0.297_{-0.002}^{+0.003} \mathrm{rad} \mathrm{d}^{-1}$.

As an additional check of the differential rotation, we tried to fit the Kepler light curve with a different set of free parameters, i.e. by allowing the spot latitudes to vary without any constraint on the differential rotation law. To save computing time, we applied this model to the Q0+Q1 dataset, which is, however, large enough to constrain the rotation period of each spot. As a result, we found that the slowly revolving spots are at high latitudes. Despite the spot latitudes being notoriously ill-defined by photometry, at least for ground-based data, the stellar surface rotates beyond any doubt in a Sun-like way.

Expectation values with 1- $\sigma$ confidence limits for some interesting parameters are given in Table 5. The reader should be aware that the estimated parameter values and their (often surprisingly small) error bars are those of the model constrained by the data. The error bars simply indicate the "elbow room" of the model, nothing more.

There is a pronounced long-term trend in the light curve. The star gets dimmer with time. To prevent any instrumental effect affecting the outcome of the photometric spot modeling, we allowed not only for offsets, but, moreover, for adjustable slopes in the seven time chunks. These slopes are considered nuisance

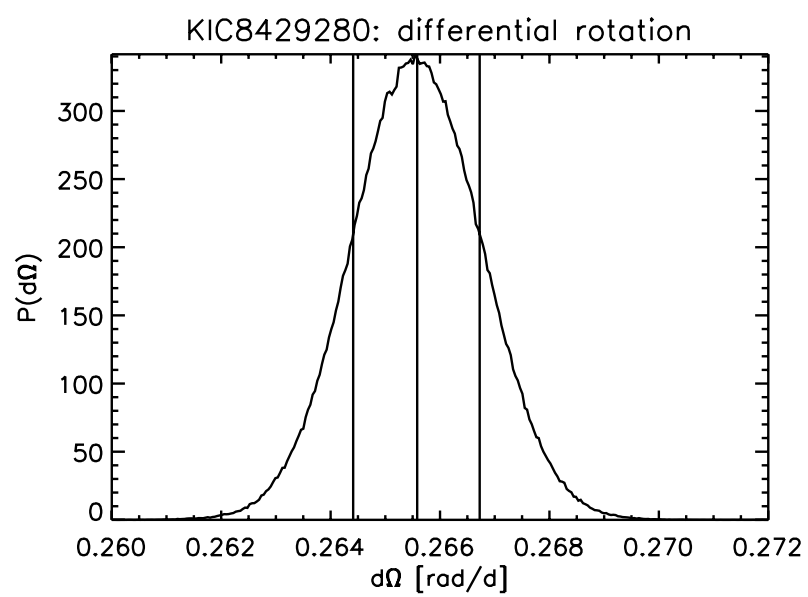

Fig. 13. The equator-to-pole differential rotation. Vertical lines mark the mean and the 68-per-cent confidence region.

Table 5. Two 7-spot solutions.

\begin{tabular}{|c|c|c|c|c|c|c|}
\hline \multicolumn{3}{|c|}{ Parameter } & \multicolumn{2}{|c|}{ 1st solution } & \multicolumn{2}{|c|}{ 2nd solution } \\
\hline \multicolumn{2}{|c|}{ inclination } & $i$ & 69.5 & $\begin{array}{l}+0.4 \\
-0.4\end{array}$ & $69: 6$ & $\begin{array}{l}+0.4 \\
-0.4\end{array}$ \\
\hline $1 \mathrm{st}$ & latitude & $\beta_{1}$ & 62.5 & $\begin{array}{l}+0.2 \\
-0.2\end{array}$ & $57: 1$ & $\begin{array}{l}+0.5 \\
-0.3\end{array}$ \\
\hline 2nd & latitude & $\beta_{2}^{\mathrm{a}}$ & -0.5 & $\begin{array}{l}+0.4 \\
-0.2\end{array}$ & -0.8 & $\begin{array}{l}+0.6 \\
-0.2\end{array}$ \\
\hline $3 \mathrm{rd}$ & latitude & $\beta_{3}$ & 62.5 & $\begin{array}{l}+0.2 \\
-0.2\end{array}$ & 57.5 & $\begin{array}{l}+0.5 \\
-0.3\end{array}$ \\
\hline 4 th & latitude & $\beta_{4}$ & $17: 3$ & ${ }_{-0.2}^{+0.2}$ & 16.5 & ${ }_{-0.2}^{+0.2}$ \\
\hline 5 th & latitude & $\beta_{5}$ & $-53: 9$ & $\begin{array}{l}+0.2 \\
-0.2\end{array}$ & $56: 7$ & $\begin{array}{l}+0.5 \\
-0.3\end{array}$ \\
\hline 6th & latitude & $\beta_{6}$ & $-8: 0$ & ${ }_{-0.2}^{+0.2}$ & 10.4 & $\begin{array}{l}+0.3 \\
-0.3\end{array}$ \\
\hline 7 th & latitude & $\beta_{7}$ & -58.9 & ${ }_{-0.2}^{+0.2}$ & $-49^{\circ} .1$ & ${ }_{-0.4}^{+0.5}$ \\
\hline $1 \mathrm{st}$ & period & $P_{1}$ & 1.20430 & $\begin{array}{l}+0.00004 \\
-0.00004\end{array}$ & 1.20390 & $\begin{array}{l}+0.00004 \\
-0.00004\end{array}$ \\
\hline 2nd & period & $P_{2}$ & 1.15793 & $\begin{array}{l}+0.00007 \\
-0.00007\end{array}$ & 1.15741 & $\begin{array}{l}+0.00009 \\
-0.00009\end{array}$ \\
\hline $3 \mathrm{rd}$ & period & $P_{3}$ & 1.20430 & $\begin{array}{l}+0.00005 \\
-0.00005\end{array}$ & 1.20430 & $\begin{array}{l}+0.00006 \\
-0.00007\end{array}$ \\
\hline 4 th & period & $P_{4}$ & 1.16298 & $\begin{array}{l}+0.00007 \\
{ }_{-0.00007}\end{array}$ & 1.16253 & $\begin{array}{l}+0.00006 \\
-0.00005\end{array}$ \\
\hline 5 th & period & $P_{5}$ & 1.19617 & $\begin{array}{l}+0.00012 \\
-0.00012\end{array}$ & 1.20345 & $\begin{array}{l}-0.00004 \\
-0.00004\end{array}$ \\
\hline 6th & period & $P_{6}$ & 1.15903 & $\begin{array}{l}+0.00005 \\
-0.00005\end{array}$ & 1.15948 & $\begin{array}{l}+0.00003 \\
-0.00003\end{array}$ \\
\hline 7 th & period & $P_{7}$ & 1.20107 & $\begin{array}{l}+0.00008 \\
-0.00007\end{array}$ & 1.19479 & $\begin{array}{l}+0.00013 \\
+0.00014\end{array}$ \\
\hline \multicolumn{2}{|c|}{ spot intensity } & $\kappa$ & 0.574 & $\begin{array}{l}+0.006 \\
-0.006\end{array}$ & 0.599 & $\begin{array}{l}+0.017 \\
{ }_{-0.015}\end{array}$ \\
\hline \multicolumn{2}{|c|}{ diff. rotation } & $d \Omega$ & 0.2656 & $\begin{array}{l}+0.0011 \\
-0.0012\end{array}$ & 0.2974 & $\begin{array}{l}+0.0016 \\
-0.0032\end{array}$ \\
\hline
\end{tabular}

Notes. Listed are expectation values and 1- $\sigma$ uncertainties. Latitudes $\beta$ are derived from the assumed law of differential rotation (Eq. (2)). Periods $P$ are given in days, the spot intensity $\kappa$ is in units of the intensity of the unspotted surface. The differential rotation $\mathrm{d} \Omega\left(\mathrm{rad} \mathrm{d}^{-1}\right)$ is the equator-to-pole value of the shear. ${ }^{(a)}$ The skewed error estimates for the latitude of the second spot are calculated under the assumption that it is located south of the equator.

parameters and analytically integrated away. The fit is, of course, tighter, with the residuals now being reduced to 2.17 mag rms. Most importantly, our main results are unaffected. The inclination is lowered by merely three degrees, the spot intensity is slightly increased to 0.635 , and the equator-to-pole differential rotation is increased somewhat to $0.269 \pm 0.003 \mathrm{rad} \mathrm{d}^{-1}$, which is between the values of the two solutions set out in Table 5 . Owing to the smallness of these changes, there is no need to be doubtful about the long-term stability of the data.

An additional improvement could be achieved by including more free parameters in the spot area evolution ansatz. The 
easiest way to do this is to substitute the sharp bend between the two slopes with a more gradual transition. This can be achieved, for example, by a linear interpolation between the values of the two slopes of the first derivative of the area-versus-time relation over some time span, which is the new free parameter. The longer this time, the smoother the bend between the two time evolution regimes. With seven free parameters more, the residuals are reduced from $2.36 \mathrm{mmag}$ to $2.25 \mathrm{mmag}$, which is, because of the large number of data points involved, formally significant, but the main outcome remains nevertheless unchanged. The inclination has decreased by a half of degree to $i=68^{\circ} .9 \pm 0.4$ and the equator-to-pole differential rotation has decreased to $0.254 \pm 0.001 \mathrm{rad} \mathrm{d}^{-1}$.

\section{Discussion}

\subsection{Is KIC 8429280 a binary?}

The constant radial velocity and the absence of secondary peaks in the CCF (Sect. 3.1) seem to exclude that KIC 8429280 is a spectroscopic binary in a short-period orbit.

However, in the WDS Catalog (Worley \& Douglass 1997) the source is classified as a close visual binary composed of two stars of equal magnitude with a separation of 0.3 and a position angle $\mathrm{PA}=22^{\circ}$. The two components are resolved only in one visual observation performed in 1988 with the 0.5 -m Nice refractor by Couteau (1990). However, although the star is present in the Tycho Double Star Catalog (Fabricius et al. 2002), no entry for the separation and position angle is present, even though in this catalog binaries with separations $\rho \geq 0$. .'2 and components of similar magnitude are normally resolved. Evidently, the Tycho instrument on-board the Hipparcos satellite, which was operational from December 1989 to February 1993 (only 1-5 years later than the Couteau observation), was unable to distinguish the components of this possible binary. As far as we know, no other hint of binarity is present in the literature.

If we consider two identical stars with the same luminosity, temperature $\left(T_{\mathrm{eff}}=5055 \mathrm{~K}\right)$, and radius $\left(R=0.88 R_{\odot}\right)$, the distance estimated from the SED increases to about $85 \mathrm{pc}$. At this distance, an apparent separation of 0 '. 3 corresponds to about $25 \mathrm{AU}$. From the third Kepler law, with a mass of about $0.9 M_{\odot}$ per each component, we can estimate a minimum orbital period $P_{\text {orb }} \approx 93$ years, assuming that the semi-major axis of the orbit is $a \approx a \sin i=25 \mathrm{AU}$. Thus, given the long orbital period, the apparent separation of the two components should have been nearly the same during the Couteaus's and Tycho observations.

Moreover, in this hypothesis we can evaluate the semiamplitude of the $R V$ curve as

$k=\frac{2 \pi}{P_{\text {orb }}} \frac{a \sin i}{\left(1-e^{2}\right)^{1 / 2}} \approx \frac{8}{\left(1-e^{2}\right)^{1 / 2}} \mathrm{~km} \mathrm{~s}^{-1}$

were we assume that $a \sin i=25 \mathrm{AU}$. An $R V$ variation should be easily detectable with observations taken a few years apart.

From all the previous considerations, it is unlikely that KIC 8429280 is a double star. However, more spectra and high angular resolution images are needed to safely exclude the presence of a stellar companion.

\subsection{Inclination}

The photometrically obtained value of the inclination, $i \simeq 69.5^{\circ}$, and the spectroscopically determined $v \sin i$ allow one to determine the radius of the star. Assuming the shortest rotational period to be associated with the equator, one arrives at $R=0.93 R_{\odot}$, which is close to the expected value (see Sect. 3.3). As the inclination depends crucially on the model assumptions, this correspondence strengthens our confidence in the reliability of our spot-modeling approach.

\subsection{Spot contrast}

In a first analysis, considering quarters Q0 and Q1 only, we identified rather large spots with a very low spot-to-photosphere contrast $(\kappa \simeq 0.8)$. After analyzing a three-times longer time span, the spot surface brightness (Fig. 10) in the Kepler photometric band amounts to the comforting value of $60 \%$ of the photospheric intensity. The corresponding temperature contrast $T_{\text {sp }} / T_{\text {ph }}$ is 0.87 .

In the case of 4 PMS stars in Orion ( 2 Myr old), Frasca et al. (2009) found a temperature contrast $T_{\mathrm{sp}} / T_{\mathrm{ph}}$ in the range $0.71-$ 0.92 . For fairly young stars $(\sim 200-400 \mathrm{Myr})$ such as $\kappa^{1}$ Cet, HD 166, and $\epsilon$ Eri, Biazzo et al. (2007) derived values of $T_{\mathrm{sp}} / T_{\mathrm{ph}} \sim 0.85$ from the simultaneous modeling of temperature (from line-depth ratio analysis) and light curves. The spots of KIC8429280 are, therefore, comparable to both those of Orion PMS and mildly active stars. However, we recall that a "spot" can be more than a single dark spot, namely an active region, which is a mixture of dark and bright surface features.

However, despite their very high precision, the Kepler magnitudes are taken in "white light", i.e. with only one very broad band. Thus, there is no possibility of constraining the spot contrast without a contemporaneous multi-band photometric or spectroscopic monitoring.

\subsection{Differential rotation}

One of the unexpected results of the present work is the degree of differential rotation of KIC 8429280. Assuming a $\sin ^{2}$ law for the latitudinal dependence, one arrives at $0.27-0.30 \mathrm{rad} \mathrm{d}^{-1}$ for the equator-to-pole difference in angular velocity. This is about five times larger than in the case of the Sun.

Barnes et al. (2005) showed that in general differential rotation increases with stellar effective temperature. The highest value reported by them for stars as cool as $\sim 5000 \mathrm{~K}, \mathrm{~d} \Omega \approx 0.2$, is that measured by Donati et al. (2003) for LQ Hya in 2000. Marsden et al. (2011) extended this dataset with values of $\mathrm{d} \Omega$ (in the range $0.08-0.45 \mathrm{rad} \mathrm{d}^{-1}$ ) measured only for stars more massive than the Sun.

For LQ Hya, which has similar stellar parameters and is rotating a little bit slower than our target (cf. Sect.3.2), Kővári et al. (2004) determined the differential surface rotation from the cross-correlation of several Doppler maps. A solar-type differential rotation law, i.e. the equator rotating faster than the poles, with $\mathrm{d} \Omega=0.022 \mathrm{rad} \mathrm{d}^{-1}$ (lap time of $\approx 280$ days) is found by the aforementioned authors. Donati et al. (2003) measured surface differential rotation in LQHya and AB Dor by means of a new Doppler imaging technique that includes among its free parameters those of a solar-type differential rotation law. Their results show that the equatorial angular velocity and the amplitude of the latitudinal shear of AB Dor and LQ Hya change on timescales of a few years. This is ascribed to the redistribution of the angular momentum inside the stars likely to be caused by the action of a non-linear hydromagnetic dynamo. In particular, they found for LQHya a decrease in the shear between years 2000 $\left(\mathrm{d} \Omega \simeq 0.20 \mathrm{rad} \mathrm{d}^{-1}\right)$ and $2001\left(0.01 \mathrm{rad} \mathrm{d}^{-1}\right)$.

HD 141943, another very young more-massive star that is still in the PMS phase, has values of $\mathrm{d} \Omega$ ranging from about 0.23 
to $0.44 \mathrm{rad} \mathrm{d}^{-1}$ in different epochs as derived by Marsden et al. (2011).

Very different values of differential rotation have also been found for HD 171488, a young ( 50 Myr) Sun. For this star, a very high solar-type differential rotation $\mathrm{d} \Omega \approx 0.4-0.5 \mathrm{rad} \mathrm{d}^{-1}$, with the equator lapping the poles every $12-16$ days, was found by both Marsden et al. (2006) and Jeffers \& Donati (2008). Much weaker values $(\mathrm{d} \Omega \approx 0.04)$ were derived instead for the same star by Järvinen et al. (2008) and Kővári et al. (2010).

A weak differential rotation $(d \Omega$ between 0.017 and $0.056 \mathrm{rad} \mathrm{d}^{-1}$ ) was found for $\epsilon$ Eri by Fröhlich (2007) from a Bayesian reanalysis of the MOST light curve (Croll 2006; Croll et al. 2006). This is a mildly active star with the same spectral type as KIC 8429280 (K2 V), but with a considerably longer rotational period $\left(P_{\text {rot }} \simeq 11.2\right.$ days $)$.

Thus, from these observations, it seems that a higher differential rotation is more frequently encountered in rapid rotators, although different indications come from previous works, mainly based on ground-based photometry, which seem to display the opposite behavior, i.e. a differential rotation decreasing with the rotation period (e.g., Messina \& Guinan 2003). The precision of the ground-based light curves does not allow to draw firm conclusions and accurate photometry from space, as well as Doppler imaging, is strongly needed to enlarge the database of differential rotation measurements that must be compared with the results of new dynamo models for stars in different evolutionary phases (including the PMS stage) and with both low and high rotation rates.

The high differential rotation that we found for KIC 8429280 disagrees with the predictions of the model of Küker et al. (2011), who instead found rather low values of $\mathrm{d} \Omega \approx 0.08$ for an (evolved) solar-mass star rotating with a period as short as 1.3 days. However, this specific model was made for a star with a remarkably different mass, effective temperature, and inner structure.

Covas et al. (2005) developed dynamo models capable of reproducing variations in surface differential rotation along the activity cycle, although they cannot produce anything as extreme as the episodes of almost rigid surface rotation $(\mathrm{d} \Omega \approx 0.0)$ as reported for LQ Hya. On the other hand, these models can reproduce variations in the differential rotation of several percent for stars with deep convective envelopes rotating ten times faster than the Sun. Lanza (2006) found that the large value of the rotation shear $(\sim 0.2)$ observed for LQ Hya in the year 2000 implies a dissipated power that is comparable to the star luminosity, but this high shear can be maintained for a time of a few years.

The value of $0.27-0.30 \mathrm{rad} \mathrm{d}^{-1}$ we derive for KIC 8429280 is the largest ever measured, to our knowledge, for a young star less massive than the Sun. The analysis of Kepler photometry acquired in subsequent epochs will enable us to detect any eventual variation in the surface differential rotation.

\section{Conclusion}

We have performed an accurate analysis of high-resolution spectra and high-precision Kepler photometry of KIC 8429280 designed to characterize the chromospheric and photospheric activity.

Thanks to the high-resolution spectra we have derived, for the first time, the astrophysical parameters, $T_{\text {eff }}, \log g,[\mathrm{Fe} / \mathrm{H}]$, rotational and heliocentric radial velocity, and lithium abundance. The lithium abundance allows us to estimate an age of about $50 \mathrm{Myr}$, that, with a mass of about $0.9 M_{\odot}$, is likely to imply a pre-main sequence stage with the star approaching the ZAMS along its radiative track.

A high chromospheric activity level was inferred from different spectral diagnostics ( $\mathrm{H} \alpha, \mathrm{H} \beta, \mathrm{Ca}$ II $\mathrm{H} \& \mathrm{~K}$, and $\mathrm{Ca}$ II IRT lines) by applying the spectral subtraction technique. Both the Balmer decrement, measured as the ratio of $\mathrm{H} \alpha$ to $\mathrm{H} \beta$ emission flux, and the flux ratio of two Ca II IRT lines are indicative of optically thick emission similar to that observed from extended facular regions.

The application of a robust spot model, based on a Bayesian approach and a Markov chain Monte Carlo method, to the highprecision Kepler photometry spanning 138 days, has allowed us to map the photospheric spots and study their time evolution. Seven spots were needed to perform a reasonable fit to the Kepler light curve, although, because of the exceptional precision of the Kepler photometry, it is impossible to reach the noise floor without increasing significantly the degrees of freedom and, consequently, the non-uniqueness of the solution. The active regions turn out to be mainly located around three latitude belts, i.e. around the star's equator and around $\pm\left(50^{\circ}-60^{\circ}\right)$, with the highlatitude spots rotating slower than the low-latitude ones.

The equator-to-pole differential rotation $\mathrm{d} \Omega \simeq 0.27 \mathrm{rad} \mathrm{d}^{-1}$ is one of the largest values ever measured for a star cooler than the Sun. This is at variance with some recent theoretical models that also predict a moderate solar-type differential rotation for very rapidly rotating main-sequence stars (Küker et al. 2011) but could fit the scenario proposed by other modelers of a higher $\mathrm{d} \Omega$, which can change along the activity cycle (Covas et al. 2005; Lanza 2006).

Acknowledgements. We are grateful to the anonymous referee for a careful reading of the paper and valuable comments. This work has been partly supported by the Italian Ministero dell'Istruzione, Università e Ricerca (MIUR) and by the Regione Sicilia which are gratefully acknowledged. J.M.-Ż acknowlegdes the Polish Ministry grant No. N N203 405139. K.B. acknowledges financial support from the INAF Postdoctoral fellowship. This research made use of SIMBAD and VIZIER databases, operated at the CDS, Strasbourg, France.

\section{References}

Allende Prieto, C., Barklem, P. S., Lambert, D. L., \& Cunha, K. 2004, A\&A, 420, 183

Alonso, R., Auvergne, M., Baglin, A., et al. 2008, A\&A, 482, L21

Barnes, T. G., \& Evans, D. S. 1976, MNRAS, 174, 489

Barnes, J. R., Collier Cameron, A., Donati, J.-F., et al. 2005, MNRAS, 357, L1

Biazzo, K., Frasca, A., Henry, G. W., Catalano, S., \& Marilli, E. 2007, ApJ, 656, 474

Biazzo, K., Frasca, A., Marilli, E., et al. 2009, A\&A, 499, 579

Bonanno, A., Elstner, D., Rüdiger, G., \& Belvedere, G. 2002, A\&A, 390, 673

Borucki, W. J., Koch, D., Basri, G., et al. 2010, Science, 327, 977

Buzasi, D. L. 1989, Ph.D. Thesis, Pennsylvania State Univ.

Cardelli, J. A., Clayton, G. C., \& Mathis, J. S. 1989, ApJ, 345, 245

Castelaz, M. W., Persinger, T., Stein, J. W. Prosser, J., \& Powell, H. D. 1991, AJ, 102,2103

Castelli, F., \& Hubrig, S. 2004, A\&A, 425, 263

Catanzaro, G., Frasca, A., Molenda-Żakowicz, J., \& Marilli, E. 2010, A\&A, 517, A3

Chester, M. M. 1991, Ph.D. Thesis, Pennsylvania State Univ.

Couteau, P. 1990, A\&AS, 83, 331

Covas, E., Moss, D., \& Tavakol, R. 2005, A\&A, 429, 657

Croll, B. 2006, PASP, 118, 1351

Croll, B., Walker, G. A. H., Kuschnig, R., et al. 2006, ApJ, 648, 607

Cutri, R. M., Skrutskie, M. F., Van Dyk, S., et al. 2003, 2MASS All Sky Catalog of point sources, NASA/IPAC Infrared Science Archive

Donati, J.-F., Collier Cameron, A., \& Petit, P. 2003, MNRAS, 345, 1187

Dorren, J. D. 1987, ApJ, 320, 756

Evans, D. S. 1967, in IAU Symp. 30, ed. A. H. Battened, \& J. F. Heard (London: Academic Press), 57

Fabricius, C., Hog, E., Makarov, V. V., et al. 2002, A\&A, 384, 180

Fekel, F. C., Bopp, B. W., Africano, J. L., et al. 1986, AJ, 92, 1150

Frasca, A., \& Catalano, S. 1994, A\&A, 284, 883 
Frasca, A., Alcalá, J. M., Covino, E., et al. 2003, A\&A, 405, 149 Frasca, A., Guillout, P., Marilli, E., et al. 2006, A\&A, 454, 301

Frasca, A., Kővári, Zs., Strassmeier, K. G., \& Biazzo, K. 2008, A\&A, 481, 229

Frasca, A., Covino, E., Spezzi, L., et al. 2009, A\&A, 508, 1313

Frasca, A., Biazzo, K., Kővári, Zs., Marilli, E., \& Çakırlı, Ö. 2010, A\&A, 518, A48

Fröhlich, H.-E. 2007, Astron. Nachr., 238, 1037

Glebocki, R., \& Gnacinski, P. 2005, The Catalogue of Rotational Velocities of Stars, ESA SP, 560, 571

Guillout, P., Klutsch, A., Frasca, A., et al. 2009, A\&A, 504, 829

Gunn, J., \& Stryker, L. L. 1983, ApJS, 52, 121

Hauschildt, P. H., Allard, F., \& Baron, E. 1999, ApJ, 512, 377

Herbig, G. H. 1985, ApJ, 289, 269

Järvinen, S. P., Korhonen, H., Berdyugina, S. V., et al. 2008, A\&A, 488, 1047

Jeffers, S. D., \& Donati, J.-F. 2008, MNRAS, 390, 635

Kass, R. E., Wasserman, L. 1996, J. Am. Stat. Association, 91, 1343

Kővári, Zs., Strassmeier, K. G., Granzer, T., et al. 2004, A\&A, 417, 1047

Kővári, Zs., Frasca, A., Biazzo, K., et al. 2010, Physics of Sun and Star Spots, ed. D. P. Choudhary, \& K. G. Strassmeier (Cambridge Univ. Press), Proc. IAU Symp., 273 [arXiv: 1010.3511]

Küker, M., \& Rüdiger, G. 1999, A\&A, 346, 922

Küker, M., Rüdiger, G., \& Kitchatinov, L. L. 2011, A\&A, 530, A48

Kurucz, R. L. 1993, A new opacity-sampling model atmosphere program for arbitrary abundances, in peculiar versus normal phenomena in A-type and related stars, ed. M. M. Dworetsky, F. Castelli, R. Faraggiana, IAU Coll., 138, ASP Conf. Ser., 44, 87

Kurucz, R. L., \& Avrett, E. H. 1981, SAO Special Rep., 391

Kurucz, R. L., \& Bell, B. 1995, Kurucz CD-ROM No. 23 (Cambridge, Mass.: Smithsonian Astrophysical Observatory)

Lanza, A. F. 2006, MNRAS, 373, 819
Lanza, A. F., Rodonò, M., \& Zappala, R. A. 1994, A\&A, 290, 861

Lanza, A. F., Rodonò, M., Pagano, I., Barge, P., \& Llebaria, A. 2003, A\&A, 403, 1135

Marsden, S. C., Donati, J.-F., Semel, M., Petit, P., \& Carter, B. D. 2006, MNRAS, 370,468

Marsden, S. C., Jardine, M. M., Ramírez Vélez, J. C., et al. 2011, MNRAS, 413, 1939

Messina, S., \& Guinan, E. F. 2003, A\&A, 409, 1017

Mikolajewska, J., \& Mikolajewski, M. 1980, Acta Astron., 30, 347

Nelson, N. J., Brown, B. P., Brun, S., Miesch, M. S., \& Toomre, J. 2011, in 217th AAS Meeting, \#155.12, BAAS, 43

Nordström, B., Mayor, M., Andersen, J., et al. 2004, A\&A, 418, 989

Pavlenko, Y. V., \& Magazzù, A. 1996, A\&A, 311, 961

Perryman, M. A. C., Lindegren, L., Kovalevsky, J., et al. 1997, A\&A, 323, L49

Press, W. H., Teukolsky, S. A., Vetterling, W. T., \& Flannery, B. P. 2007, Numerical Recipes, 3rd ed. (Cambridge: Cambridge Univ. Press)

Prugniel, P., \& Soubiran, C. 2001, A\&A, 369, 1048

Schwarz, G. 1978, The Annals of Statistics, 6, 461

Sestito, P., \& Randich, S. 2005, A\&A, 442, 615

Siess, L., Dufour, E., \& Forestini, M. 2000, A\&A, 385, 593

Sing, D. K. 2010, A\&A, 510, A21

Soderblom, D. R., Jones, B. F., \& Balachandran, S., et al. 1993, AJ, 106, 1059

Stetson, P. B. 2000, PASP, 112, 925

Strassmeier, K. G., Rice, J. B., Wehlau, W. H., Hill, G. M., \& Matthews, J. M. 1993, A\&A, 268, 671

Udry, S., Mayor, M., \& Queloz, D. 1999, Precise Stellar Radial Velocities, ed. J. B. Hearnshaw, \& C. D. Scarfe, IAU Colloq., 170, ASP Conf. Ser., 185, 367

Voges, W., Aschenbach, B., Boller, T., et al. 1999, A\&A, 349, 389

Voges, W., Aschenbach, B., Boller, T., et al. 2000, IAU Circ., 7432

Worley, C. E., \& Douglass, G. G. 1997, A\&AS, 125, 523 